\title{
Enhancement of Transactivation Activity of Rta of Epstein-Barr Virus by RanBPM
}

\section{Li-Kwan Chang ${ }^{1 *}$, Shih-Tung Liu $^{2}$, Chung-Wen Kuo ${ }^{2}$, Wen-Hung Wang ${ }^{2}$, Jian-Ying Chuang ${ }^{2}$, Elisabetta Bianchi ${ }^{3}$ and Yi-Ren Hong ${ }^{4}$}

${ }^{1}$ Institute of Microbiology and Biochemistry and Department of Biochemical Science and Technology, National Taiwan University, No.1, Sec. 4, Roosevelt Rd., Taipei, 10617, Taiwan

${ }^{2}$ Molecular Genetics Laboratory, Department of Microbiology and Immunology, Chang-Gung University, 259, Wen-Hwa 1st Rd., Kwei-Shan, Taoyuan, 333, Taiwan

${ }^{3}$ Laboratory of Immunoregulation, Department of Immunology, Institut Pasteur, 25 rue du Dr. Roux, 75724 Paris, France

${ }^{4}$ Graduate Institute of

Biochemistry, Kaohsiung

Medical University, 100,

Shih-Chuan 1st Rd.,

Kaohsiung, 807, Taiwan

Received 7 January 2008;

received in revised form

1 April 2008;

accepted 1 April 2008

Available online

10 April 2008

Edited by J. Karn
Epstein-Barr virus (EBV) expresses the immediate-early protein Rta to activate the transcription of EBV lytic genes and the lytic cycle. We show that RanBPM acts as a binding partner of Rta in yeast two-hybrid analysis. The binding was confirmed by glutathione-S-transferase pull-down assay. A coimmunoprecipitation experiment and confocal microscopy revealed that RanBPM and Rta interact in vivo and colocalize in the nucleus. The interaction appears to involve the SPRY domain in RanBPM and the region between amino acid residues 416 to 476 in Rta. The interaction promotes the transactivation activity of Rta in activating the transcription of BMLF1 and p21 in transient transfection assays. Additionally, RanBPM interacts with SUMO-E2 (Ubc9) to promote sumoylation of Rta by SUMO-1. This fact explains why the expression of RanBPM enhances the transactivation activity of Rta. Taken together, the present results indicate a new role of RanBPM in regulating a viral protein that is critical to EBV lytic activation.

(C) 2008 Elsevier Ltd. All rights reserved.
${ }^{*}$ Corresponding author. E-mail address: changlk@ntu.edu.tw.

Abbreviations used: EBV, Epstein-Barr virus; SUMO, small ubiquitin-like modifier; E2, ubiquitin carrier protein ligase; E3, ubiquitin-protein isopeptide ligase; TPA, 12-O-tetradecanoylphorbol 13-acetate; GST, glutathione $S$-transferase; RRE, Rta-response element; MG132, $\mathrm{N}$-carbobenzoxy-leucyl-leucyl-leucinal; GFP, green fluorescent protein; CMV, cytomegalovirus.
Keywords: Epstein-Barr virus; Rta; RanBPM 
act alone to activate a specific set of lytic genes. For example, transcription of BLLF1 and BMLF1 is activated by Rta but not Zta. 5,6 This fact explains why EBV cannot complete its lytic cycle without Rta. ${ }^{6}$ An earlier work established that the N-terminal 320 amino acid region in Rta contains a DNAbinding domain, which binds to a $17 \mathrm{bp}$ conserved sequence called the Rta-response element (RRE) to activate transcription. ${ }^{7}$ Furthermore, Rta often interacts with cellular proteins. The outcomes of these interactions may affect the function of Rta or the cell. For example, the interaction with Ubc 9 and PIAS1 causes Rta sumoylation, which increases the transactivation activity of Rta. ${ }^{8}$ The interaction with $\mathrm{Rb}$ results in the release of E2F1 and cell-cycle progression. ${ }^{9-11}$ Moreover, Rta interacts with Sp1 via the Sp1-binding protein MCAF1. This interaction enhances the transcription of BRLF1, BNLF1, p16 and $p 21{ }^{12}$ Rta is known to activate the transcription of BZLF1 via the ZII region in the promoter, which contains an ATF1/2 site. ${ }^{13}$ Since Rta does not bind directly to this region, Rta may indirectly influence the capacity of ATF1/2 to activate the transcription by inducing ATF2 phosphorylation. ${ }^{13}$ Rta has been demonstrated to interact with TSG101 to activate the expression of EBV late genes. ${ }^{14}$

RanBPM is a $90 \mathrm{kDa}$ protein that interacts with Ran nuclear-cytoplasmic transport protein to affect protein shuttling between the cytoplasm and the nucleus. ${ }^{15,16}$ This protein contains an SPRY domain, which binds to the TrkA receptor, ${ }^{17}$ hDectin- $1 \mathrm{E}^{18}$ $\mathrm{L} 1^{19}$ and Axl/Sky receptor tyrosine kinases. ${ }^{20}$ Additionally, RanBPM interacts with many cellular receptors, including the androgen receptor, the glucocorticoid receptor and the thyroid hormone receptor, ${ }^{21,22}$ enhancing their transcriptional activities. RanBPM interacts also with various signaling proteins, including the Met receptor, Mirk, p75NTR and integrin LFA-1, to regulate signaling pathways. ${ }^{23-25}$ By inhibiting the ubiquitination of p73 $\alpha$, RanBPM stabilizes $\mathrm{p} 73 \alpha$, and increases the proapototic activity of the protein. ${ }^{26}$ This work demonstrates that RanBPM interacts with Rta to enhance the sumoylation and transactivation activity of Rta.

\section{Results}

\section{Interaction between RanBPM and Rta in yeast}

The yeast two-hybrid screening assay was performed in yeast strain YRG2 with a bait plasmid, pR476, 8,27 which encodes a fusion protein that contains a Gal4 DNA-binding domain that is fused with the N-terminal 476 amino acids of Rta. After the screening of about $6 \times 10^{5}$ colonies that were transformed with a human testes cDNA library, 56 plasmids were found to encode a protein that interacts with RN476. One of these plasmids encoded RanBPM. The interaction was further verified in the strain YRG2 that was cotransformed with pR476 and the cDNA clone that encodes RanBPM.

\section{Binding of Rta to RanBPM in vitro}

The glutathione-S-transferase (GST)-pull-down assay was performed to investigate whether Rta interacts with RanBPM in a context other than yeast. Glutathione-Sepharose beads were added to the lysate that was prepared from Escherichia coli transformed with a plasmid that expresses GST and GSTRanBPM. After washing, the beads were added to a lysate from P3HR1 cells that were transfected with an expression plasmid for Rta (pCMV-R). Proteins that were bound to the beads were eluted and analyzed by immunoblotting with anti-Rta antibody. The results indicated that Rta in the lysate (Fig. 1, lane 1) was retained by GST-RanBPM- (Fig. 1, lane 3) but not GST-glutathione-Sepharose beads (Fig. 1, lane 2). A similar assay demonstrated that His-tagged Rta in the E. coli lysate (Fig. 1, lane 4) was retained also by GST-RanBPM- (Fig. 1, lane 6) but not by GST-glutathione-Sepharose beads (Fig. 1, lane 5), confirming that Rta interacts directly with RanBPM.

\section{Interaction between Rta and RanBPM in vivo}

293T cells were cotransfected with pCMV-R and pEGFP-RanBPM. Cell lysates were then prepared at $24 \mathrm{~h}$ after transfection. Immunoblot analysis revealed that Rta was present in the lysate (Fig. 2a, lane 1), immunoprecipitated by anti-Rta antibody (Fig. 2a, lane 3) and coimmunoprecipitated with green fluorescent protein (GFP)-RanBPM by antiGFP antibody (Fig. 2a, lane 4). However, Rta was not immunoprecipitated by anti-IgG antibody (Fig. 2a, lane 2). Additionally, immunoblot analysis with anti-GFP antibody revealed the presence of GFPRanBPM in the lysate (Fig. 2a, lane 5). Both anti-Rta

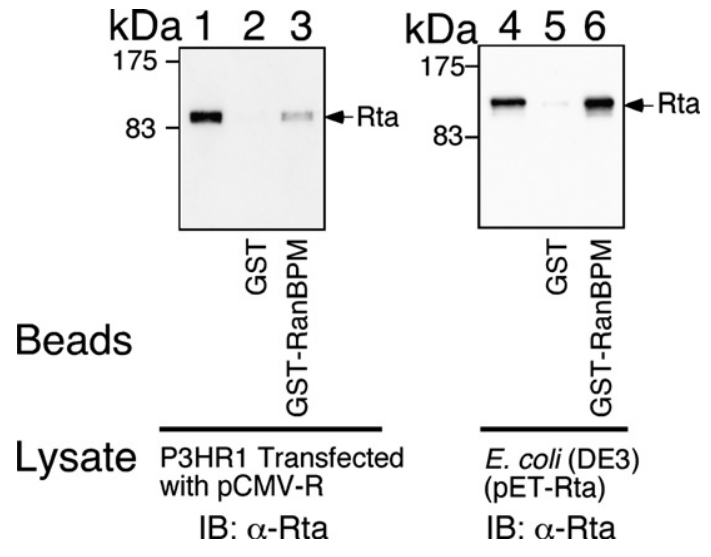

Fig. 1. Interaction between Rta and RanBPM in vitro. Lysate prepared from P3HR1 cells that had been transfected with pCMV-R (lanes 1-3) or from E. coli BL21(DE3) (pET-Rta) (lanes 4-6) was added to GST- (lanes 2 and 5) and GST-RanBPM- (lanes 3 and 6) glutathione-Sepharose beads. Lanes 1 and 4 were loaded with $5 \%$ of the indicated proteins in the cell lysate. Proteins on the beads were finally analyzed by immunoblotting (IB) with anti-Rta antibody (lanes 1-6). 
(a)

(b)
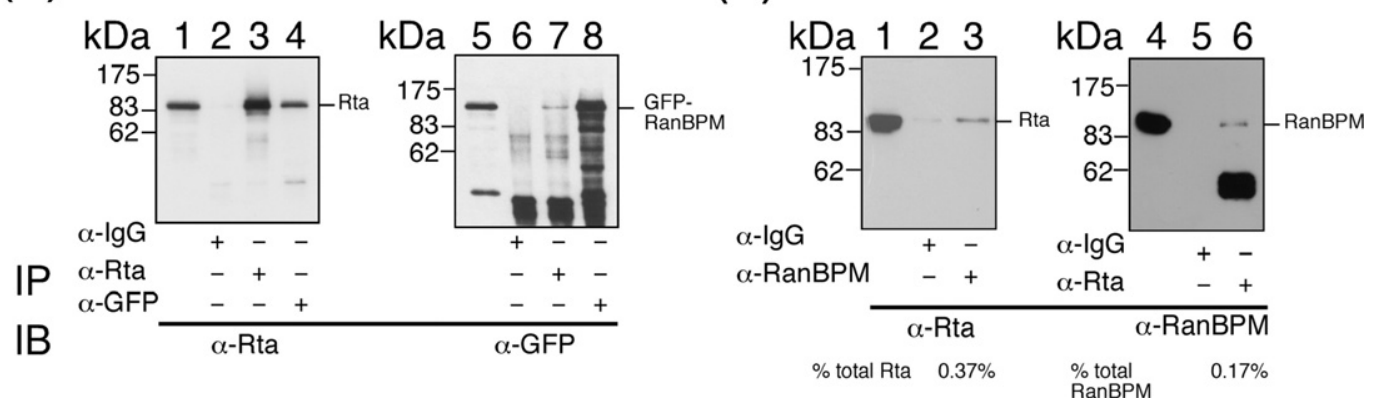

Fig. 2. Interaction between Rta and RanBPM in vivo. (a) Anti-Rta and anti-GFP antibody were added to the lysates from 293T cells that were transfected with pCMV-R and pEGFP-RanBPM. Lanes 1 and 5 were loaded with 5\% of the cell lysate. Proteins immunoprecipitated (IP) with anti-Rta antibody (lanes 3 and 7), anti-GFP antibody (lanes 4 and 8) or antiIgG (lanes 2 and 6), were detected by immunoblotting (IB) with anti-Rta antibody (lanes 1-4) and anti-GFP antibody (lanes 5-8). (b) P3HR1 cells were treated with TPA and sodium butyrate for $24 \mathrm{~h}$ to induce the EBV lytic cycle. Lanes 1 and 5 were loaded with $3 \%$ of the protein in the cell lysate. Proteins in the cell lysate were immunoprecipitated with antiRanBPM (lane 3), anti-Rta (lane 6) or anti-IgG (lanes 2 and 5) antibodies. Immunoblot analysis was performed with antiRta (lanes 1-3) and anti-RanBPM (lanes 4-6) antibodies. The percentages of Rta and RanBPM in the cell lysate that were immunoprecipitated were determined using Image J software (Wayne Rasband, National Institutes of Health, USA) to quantify the proteins in a cell extract coimmunoprecipitated with Rta or RanBPM.

antibody and anti-GFP antibody immunoprecipitated GFP-RanBPM (Fig. 2a, lanes 7 and 8). Yet, antiIgG antibody did not immunoprecipitate GFPRanBPM (Fig. 2a, lane 6). In addition, P3HR1 cells were treated with 12-O-tetradecanoylphobol-13acetate (TPA) and sodium butyrate to induce the EBV lytic cycle. After culturing the cells for $24 \mathrm{~h}$, a coimmunoprecipitation study revealed that Rta in the cell lysate was coimmunoprecipitated by antiRanBPM antibody (Fig. 2b, lane 3); RanBPM, by anti-Rta antibody (Fig. 2b, lane 6), indicating that endogenous Rta and RanBPM interact.

\section{Mapping the Rta-RanBPM interaction region in Rta}

GFP-RanBPM fusion protein was deleted to delineate the regions in RanBPM that interact with Rta. Plasmids that encode GFP-RanBPM, GFP-RanBPM209, GFP-RanBPM-334, and GFP-SPRY (Fig. 3a) were cotransfected with pCMV-R into 293T cells. An empty vector, pEGFP-C1, that expresses GFP was used as a negative control. Immunoblotting revealed that GFP-RanBPM and GFP-RanBPM-209 were present in the lysate and coimmunoprecipitated by anti-Rta antibody (Fig. 3b, lanes 3 and 4). GFP and GFP-RanBPM-334, although detectable in the lysate (Fig. 3b, lanes 1 and 5), were not coimmunoprecipitated by anti-Rta antibody (Fig. 3b, lanes 1 and 5), suggesting that the region between amino acid residues 209 and 333 in RanBPM, which contains an SPRY domain, ${ }^{28}$ interacts with Rta. Additionally, this work found that a GFP-fusion protein that contains the SPRY domain could be coimmunoprecipitated by anti-Rta antibody (Fig. 3b, lane 2), confirming the interaction between the SPRY domain and Rta. A study was performed also with deleted Rta to define the region in Rta that binds to RanBPM (Fig. 3c). These Rta proteins were expressed in the cells and were detected in the lysate by immunoblotting with anti-Rta (Fig. 3d, lanes 2-6) and antiFlag antibody (Fig. 3d, lanes 7-10). GFP-RanBPM was coimmunoprecipitated with full-length Rta, R-DN190, R-DN290, R-DN360 and R-DN415 by anti-Rta antibody and detected with anti-GFP antibody (Fig. 3d, lanes 1-6). GFP-RanBPM was coimmunoprecipitated with R-DN415, but not R-DC416, by anti-Flag antibody (Fig. 3d, lanes 7-10), indicating that the region in Rta that binds to RanBPM is located between amino acid residues 416 and 605. Since our yeast two-hybrid screening assay had established that the N-terminal 476 amino acids of Rta interact with RanBPM, the binding region of RanBPM to Rta is defined as the region between amino acids 416 and 476

\section{Colocalization of Rta and RanBPM in the nucleus}

P3HR1 cells were transfected with pEGFP-Rta to study the subcellular location of RanBPM and Rta. A confocal microscopic study revealed that RanBPM was present in the nucleus in P3HR1 cells (Fig. 4a-c) and that transfection of pEGFP-Rta caused an accumulation of RanBPM in nuclear dots (Fig. 4a-g), where it colocalized with Rta (Fig. 4a-h). On the other hand, RanBPM did not colocalize with GFP (Fig. 4a-d). In addition, P3HR1 cells were transfected with pR-DC416 and pR-DN415 to examine the colocalization of deleted Rta and RanBPM. RanBPM was colocalized with R-DN415 (Fig. 4b-1), but not with R-DC416 (Fig. 4b-h), in the nucleus. In a negative control, colocalization between RanBPM and Rta was not observed in P3HR1 cells that were transfected with pCMV-3 (Fig. 4b-d). Quantitative analysis of the merged confocal images with Zeiss LSM Image Browser software confirmed the colocalization of Rta and RanBPM (Fig. 4c). 
(a)

(b)
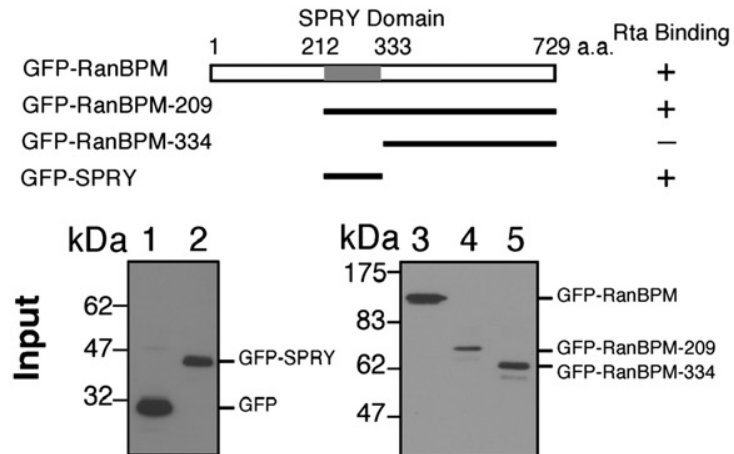

IB: $\alpha$-GFP
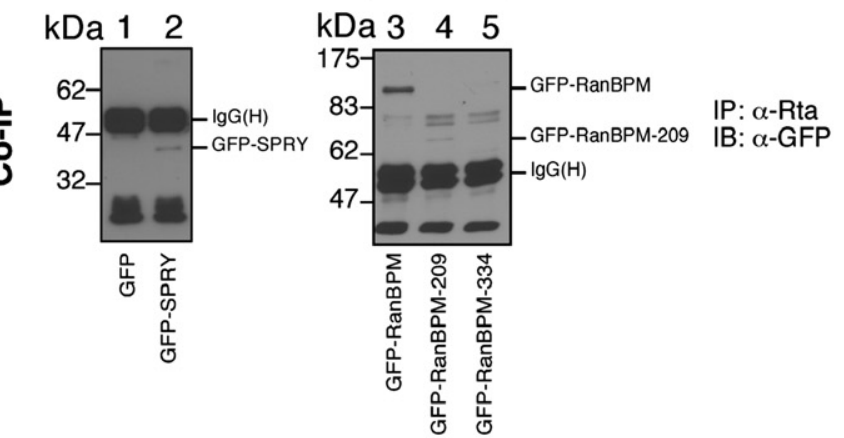

(c)

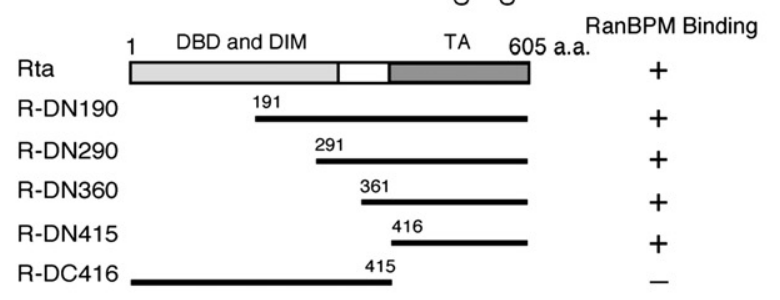

(d)
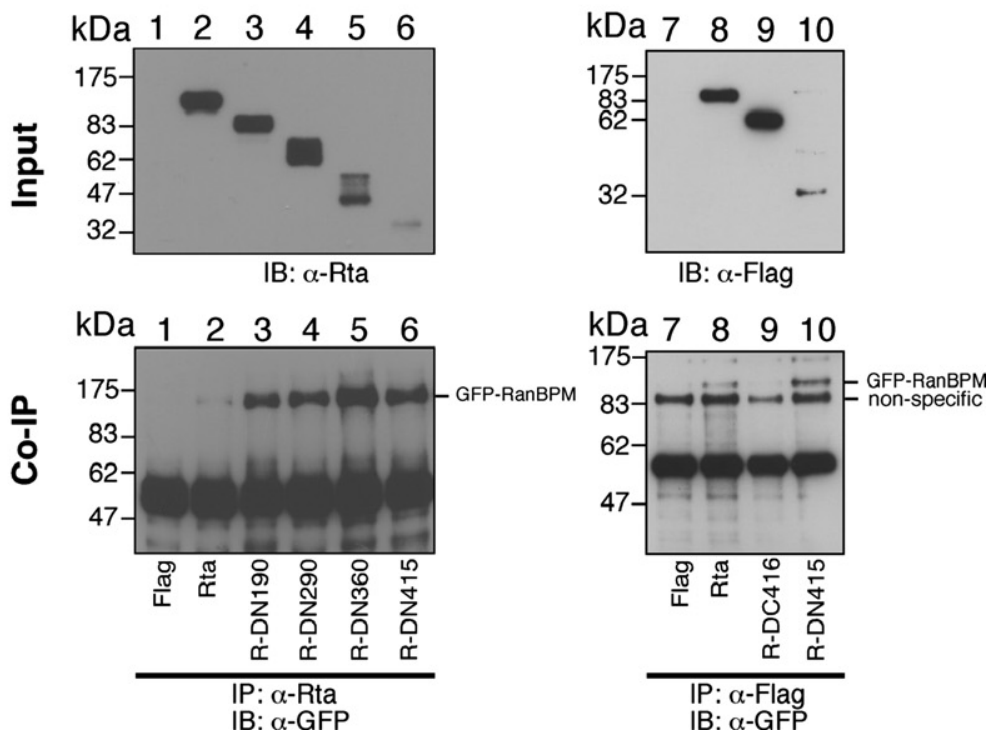

Fig. 3. Mapping the interaction domains of RanBPM and Rta. Plasmids that express deleted GFPRanBPM (a) including pEGFPRanBPM (lane 3), pEGFP-RanBPM209 (lane 4), pEGFP-RanBPM-334 (lane 5), pEGFP-SPRY (lane 2) or pEGFP-C1 (lane 1) were cotransfected with pCMV-R into 293T cells. The input, representing $5 \%$ of GFP-fusion proteins in the cell lysates, were detected by anti-GFP antibody. Proteins in the cell lysate were coimmunoprecipitated (IP) with anti-Rta antibody and analyzed by immunoblotting (IB) using antiGFP antibody. Samples in lanes 1, 2 and 3-5 were separated by SDSPAGE with $9 \%$ and $10 \%$ polyacrylamide gel, respectively. (b), Deletion mutants of Rta were used to analyze the region of Rta that interacts with RanBPM (c). 293T cells were cotransfected with pEGFP-RanBPM and Rta (lanes 2 and 8), R-DN190 (lane 3), R-DN290 (lane 4), R-DN360 (lane 5), R-DN415 (lanes 6 and10) and R-DN416 (lane 9). Lysate was then prepared and $5 \%$ of the indicated protein was loaded as an input control and detected by immunoblotting using anti-Rta and anti-Flag antibodies (d). Proteins in the lysate were then immunoprecipitated with antiRta or anti-Flag antibodies and detected by immunoblotting with antiGFP antibody (d). Plasmid pTag2A, an empty vector, was used as a negative control (lanes 1 and 7). IgG $(\mathrm{H})$, the heavy chain of IgG; DBD, DNA-binding domain; DIM, dimerization domain; TA, transactivation domain

\section{Effect of RanBPM on Rta sumoylation}

Since proteins that interact with RanBPM are frequently conjugated by SUMO ${ }^{29-33}$ we investigated whether the interaction between RanBPM and Rta affects the sumoylation of Rta. P3HR1 cells were transfected with pCMV-R, pCR-SUMO1, pEGFPRanBPM, pEF-PIASx $\beta$, and pcDNA-Ubc9. In input controls, the level of $\alpha$-tubulin, Rta, GFP-RanBPM, PIASx $\beta$ and HA-Ubc9 expression was determined by immunoblotting with anti- $\alpha$-tubulin, anti-Rta, anti-RanBPM and anti-HA antibodies, respectively (Fig. 5a, lanes 1-7). After SUMO-conjugated proteins were immunoprecipitated with anti-Flag antibody, bands of sumoylated Rta were detected by immunoblotting with anti-Rta antibody. Previous 
(a)
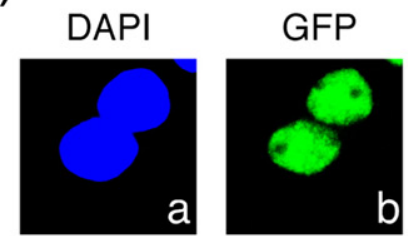

DAPI

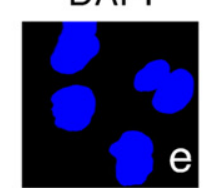

GFP-Rta

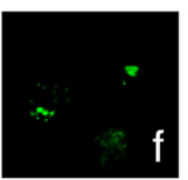

(b)
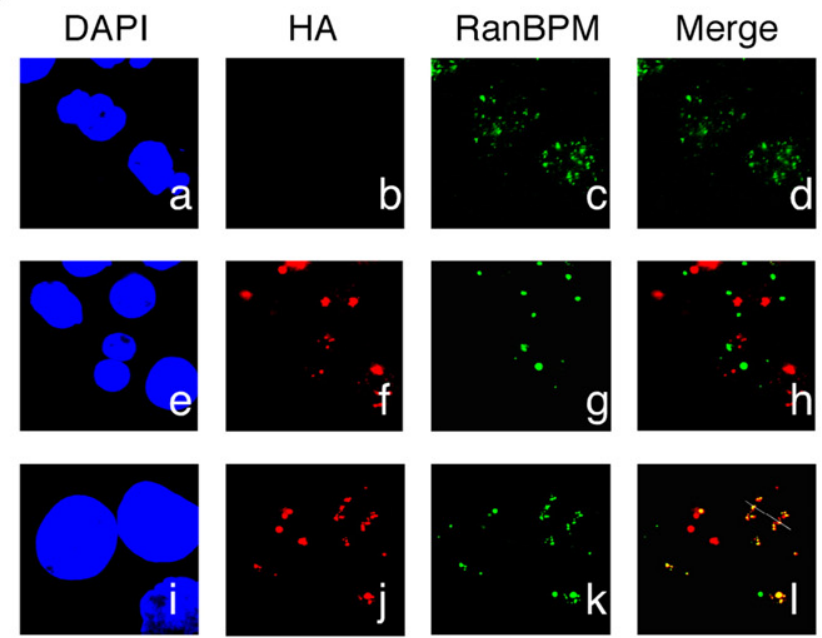

R-DN415

(c)

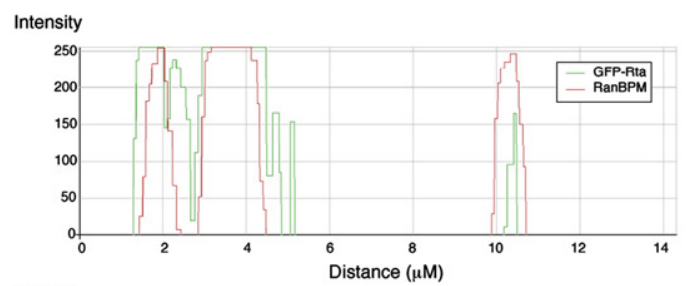

Intensity

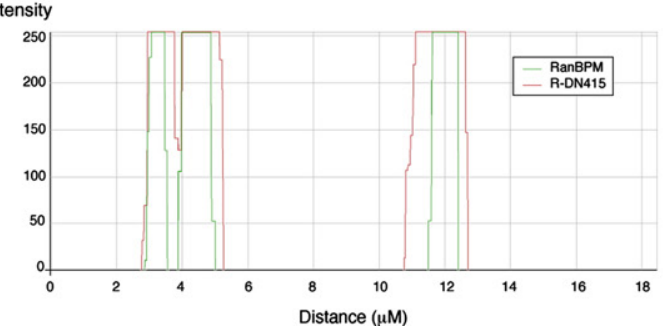

studies showed that the detectable level of SUMO-1 conjugated proteins increased in the presence of 5$10 \mu \mathrm{M}$ of MG132. ${ }^{33-35}$ Under the conditions, the amount of sumoylated Rta becomes abundant and further enhancement of Rta sumoylation by SUMO E3 ligases was difficult to detect. Therefore, in this study, we decreased the concentration of MG132 to $0.5 \mu \mathrm{M}$. Under the experimental conditions, Rta sumoylation was undetected after cotransfecting pCR-SUMO-1 and pCMV-R into P3HR1 cells (Fig. $5 a$, lane 2). However, over-expression of GFP-
Fig. 4. Indirect immunofluorescence analysis. P3HR1 cells were transfected with an empty vector (pEGFP-C1) (a, a-d), pEGFP-Rta (a, e-h), pHA-R-DC416 (b, e-h), pHA-R-DN415 (b, i-l), or pCMV-3 (b, a-d). Cells were incubated with monoclonal anti-RanBPM antibody (a, c and g; b, c, g, and k) or polyclonal anti-HA antibody (b, b, $f$ and $j$ ) and observed under a confocal laserscanning microscope. Staining with 4'-6-diamidino-2-phenylindole revealed the positions of the nucleus. (d, h and l) are merged images. Yellow color in the merged images was analyzed by the generation of a white line scan to quantify the fluorescence intensity with ZEISS LSM Image Browser software (c).

RanBPM increases Rta sumoylation, as shown by the appearance of a sumoylated Rta band by immunoblotting (Fig. 5a, lane 3). Furthermore, overexpressing Ubc9 or PIASx $\beta$ did not cause high levels of Rta sumoylation (Fig. 5a, lanes 4 and 5), which were instead seen when both proteins were over-expressed together (Fig. 5a, lane 7). Similarly, co-expressing Ubc9 and GFP-RanBPM markedly increased the amount of sumoylated Rta (Fig. 5a, lane 6); the level of Rta sumoylation appeared to be higher than that induced by Ubc9 and PIASx $\beta$ (Fig. 
(a)

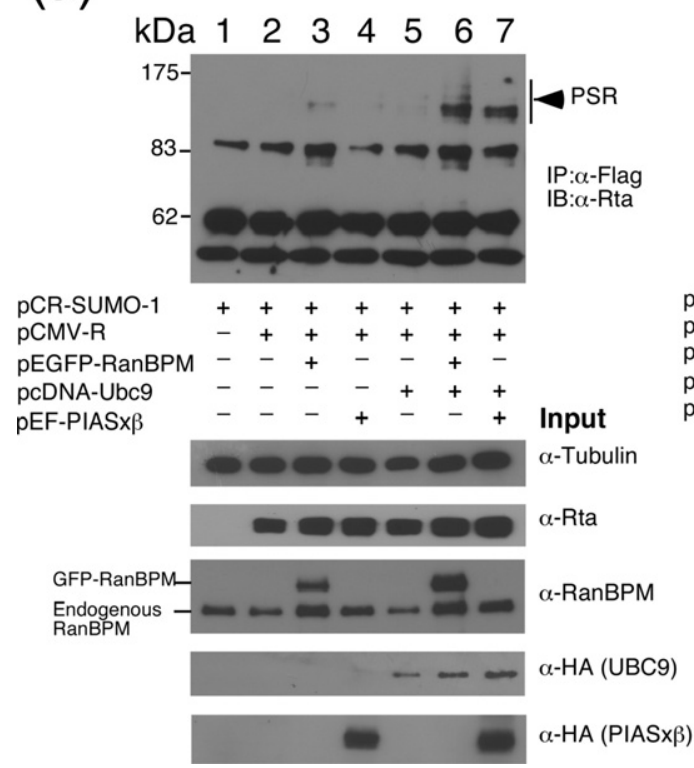

(b)

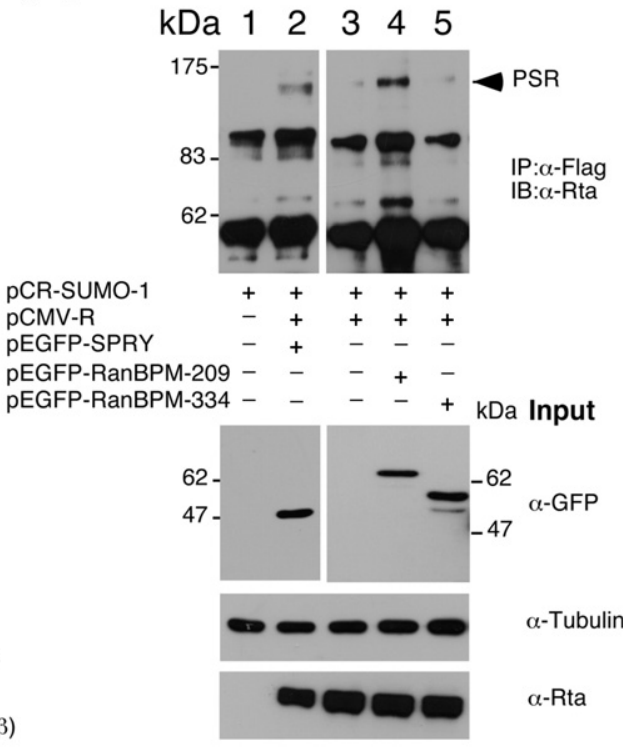

Fig. 5. Enhancement of Rta sumoylation by RanBPM. P3HR1 cells were transfected with pCR-SUMO-1, pCMV-R, pEF-PIASx $\beta$ or pcDNA-Ubc9 and treated with $0.5 \mu \mathrm{M}$ MG132. Proteins that were conjugated by Flag-tagged SUMO-1 were immunoprecipitated (IP) with anti-Flag antibody and detected by immunoblotting (IB) using anti-Rta antibody. The input control was loaded with $2.5 \%$ of the cell lysates and proteins were detected by immunoblotting with anti- $\alpha$-tubulin, anti-Rta, anti-RanBPM, and anti-HA antibodies (a). In addition, P3HR1 cells were transfected with pCR-SUMO-1, pCMV-R, pEGFP-RanBPM-209, pEGFP-RanBPM-334 or pEGFP-SPRY and treated with $0.5 \mu$ M of MG132. Similarly, proteins that were conjugated by Flag-tagged SUMO-1 were immunoprecipitated (IP) with anti-Flag antibody and detected by immunoblotting (IB) using anti-Rta antibody. The input control was loaded with $2.5 \%$ of the cell lysates and proteins were detected by immunoblotting with anti- $\alpha$-tubulin, anti-Rta and anti-GFP antibodies (b). PSR, polysumoylated Rta.

5a, lane 7). In addition, P3HR1 cells were cotransfected with pCR-SUMO-1, pCMV-R, and RanBPM deletion mutants including pEGFP-RanBPM209, pEGFP-RanBPM-334, or pEGFP-SPRY. In input control, the expression of $\alpha$-tubulin, Rta and GFP-fusion proteins were detected with anti- $\alpha$-tubulin, anti-Rta and anti-GFP antibodies, respectively (Fig. 5b). SUMO-1 conjugated Rta was detected by immunoprecipitation with anti-Flag antibody in the presence of $0.5 \mu \mathrm{M}$ MG132. Over-expression of GFP-RanBPM209 and GFP-SPRY resulted in the enhancement of Rta sumoylation (Fig. 5b, lanes 2 and 4); whereas over-expressing GFP-RanBPM-334 did not cause increased SUMO-1 conjugation to Rta (Fig. 5b, lane 5), further confirming that the SPRY domain of RanBPM is necessary to promote Rta sumoylation.

\section{Interaction between RanBPM and Ubc9}

We further investigated whether RanBPM interacts with Ubc9 to elucidate further the involvement of RanBPM in protein sumoylation. A lysate from $293 \mathrm{~T}$ cells that were cotransfected with pEGFPRanBPM and pcDNA-Ubc9 was prepared $24 \mathrm{~h}$ after transfection (Fig. 6a). The presence of GFP-RanBPM in the lysate was confirmed by immunoblot analysis with anti-GFP antibody (Fig. 6a, lane 1). GFPRanBPM was immunoprecipitated by anti-GFP antibody (Fig. 6a, lane 3) and coimmunoprecipitated with HA-Ubc9 by anti-HA antibody (Fig. 6a, lane 4). GFP-RanBPM was undetected when anti-IgG anti- body was added to the immunoprecipitation reaction (Fig. 6a, lane 2). HA-Ubc9 was also immunoprecipitated by the anti-HA antibody (Fig. 6a, lane 7) and coimmunoprecipitated with GFP-RanBPM by anti-GFP antibody (Fig. 6a, lane 8). However, HA-Ubc9 was not immunoprecipitated by anti-IgG antibody (Fig. 6a, lane 6). In addition, P3HR1 cells untreated (Fig. 6b, a-d) or treated with sodium butyrate (Fig. 6b, e-h) to induce the EBV lytic cycle were used to examine the subcellular location of RanBPM and Ubc9. A confocal microscopy study showed that RanBPM colocalizes with Ubc9 in the nucleus both in the absence (Fig. $6 \mathrm{~b}-\mathrm{d}$ ) and in the presence of Rta (Fig. 6b-h). Quantitative analysis of the merged confocal images with Zeiss LSM Image Browser software confirmed the colocalization of RanBPM and Ubc9 (Fig. 6c).

\section{Enhancement of transactivation activity of Rta by RanBPM}

Rta is known to bind to RRE in a promoter to activate transcription or interact with MCAF1 to enhance Sp1-mediated transcription. ${ }^{12}$ This study performed transient transfection to determine whether the interaction between Rta and RanBPM affects these two types of transcription. As expected, in EBV-negative Akata cells, transfection of pCMV-R activated luc transcription from pRRE and pBMLF1luc, two reporter plasmids that contain a promoter with an RRE sequence, up to 158-fold and 4.3-fold, 
(a)

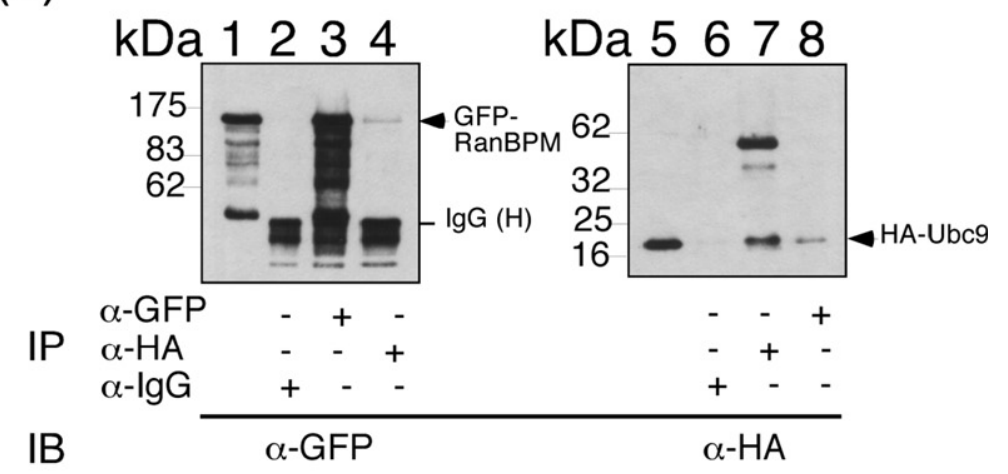

(b)

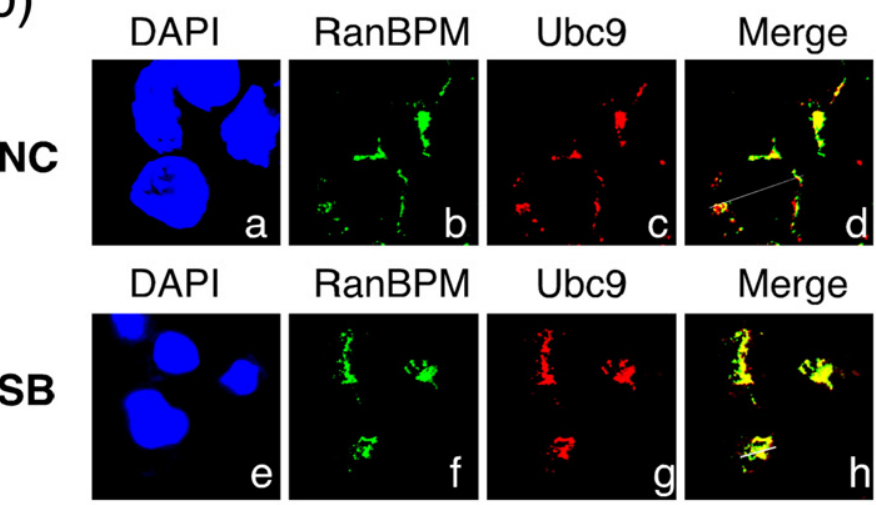

(c)
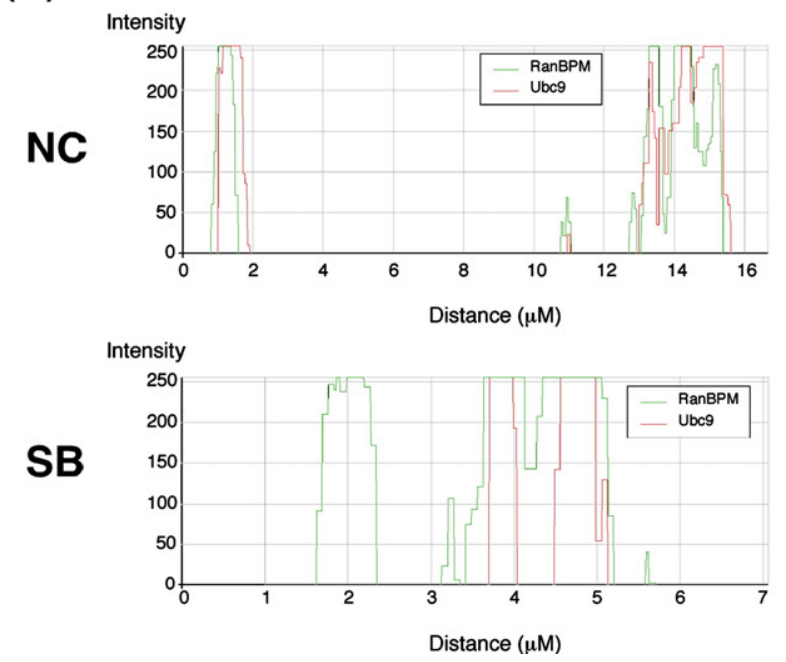

respectively (Fig. 7a and b). The promoter activity increased by 288-fold and 10.4-fold, respectively (Fig. $7 \mathrm{a}$ and $\mathrm{b}$ ) when the cells were cotransfected with pEGFP-RanBPM and pCMV-R. In the absence of pCMV-R, pEGFP-RanBPM did not activate the transcription (Fig. 7a and b), demonstrating that RanBPM promotes the transactivation activity of Rta. To determine whether RanBPM affected Sp1mediated transcription, similar experiments were performed using two reporter plasmids that contain a promoter with Sp1 binding sites, pSp1-luc and pp21-luc. As expected, transfecting pCMV-R activated pSp1-luc and pp21-luc by 150-fold and 65-fold,
Fig. 6. Interaction between RanBPM and SUMO E2 (Ubc9). (a) $293 \mathrm{~T}$ cells were cotransfected with pEGFP-RanBPM and pcDNA-Ubc9. Coimmunoprecipitation was performed with anti-GFP antibody (lanes 3 and 8) and anti-HA antibody (lanes 4 and 7). Immunoprecipitation (IP) was also performed using anti-IgG antibody (lanes 2 and 6). Immunoblotting (IB) was performed with anti-GFP (lanes 1-4) and anti-HA antibodies (lanes 5-8). Lanes 1 and 5 were loaded with 5\% of the cell lysate. $\operatorname{IgG}(\mathrm{H})$, the heavy chain of IgG. (b) In addition, P3HR1 cells were treated with sodium butyrate (SB) to induce EBV lytic cycle. Cells were incubated with monoclonal anti-RanBPM antibody (b and $\mathrm{f}$ ) and polyclonal anti-Ubc9 antibody (c and g), then observed under a confocal laser scanning microscope. Staining with 4'-6-diamidino-2-phenylindole revealed the positions of the nucleus. $\mathrm{d}$ and $\mathrm{h}$ are merged images. Yellow color in the merged images was analyzed by the generation of a white line scan to quantify the fluorescence intensity according to ZEISS LSM Image Browser software (c). NC, P3HR1 cells untreated with SB. respectively (Fig. 7c and d); the luciferase activity increased by 234-fold and 121-fold, respectively (Fig. $7 \mathrm{c}$ and $\mathrm{d})$, when the cells were cotransfected with pEGFP-RanBPM. Additionally, plasmid pLG5, a reporter plasmid that contains a promoter with five copies of Gal4-binding sites, was utilized to determine how Ubc9, SUMO-1 and RanBPM influence the capacity of Rta to transactivate the promoter. In EBVnegative Akata cells, cotransfecting pLG5 and pMRta, which expressed Gal4-Rta, activated the Gal4 promoter (Fig. 8). In the presence of Ubc9, SUMO-1 and RanBPM, the capacity of Gal4-Rta to activate the promoter increased 3.1-fold, 2.5-fold and 3.3-fold, 
(a)

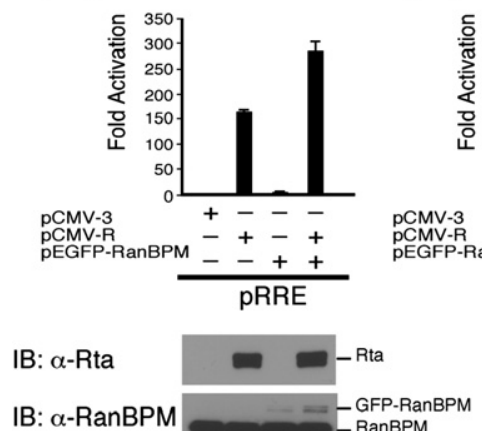

(b)

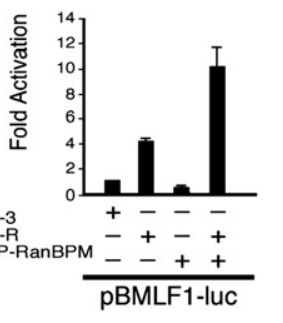

(c)

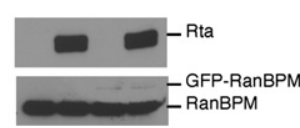

(d)
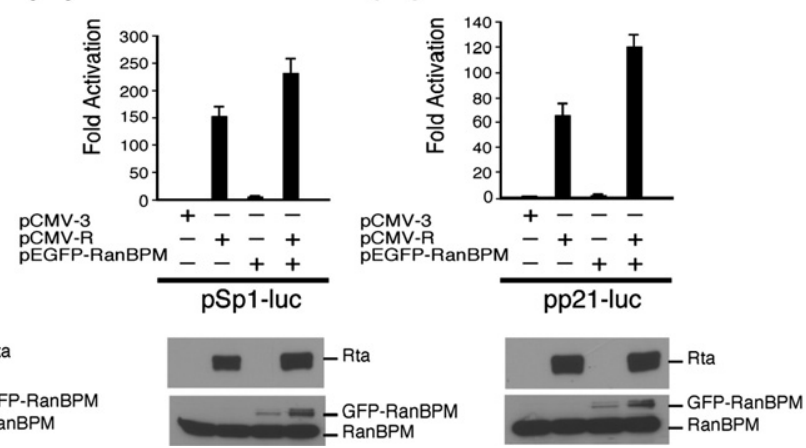

Fig. 7. RanBPM enhances the transactivation activity of Rta in EBV-negative Akata cells. Four reporter plasmids pRRE (a), pBMLF1-luc (b), pSp1-luc (c) and pp21-luc (d) - were cotransfected with pCMV-3, pCMV-R or pEGFP-RanBPM into EBV-negative Akata cells. Luciferase activity that was exhibited by the cells was examined at $24 \mathrm{~h}$ after transfection. Each transfection experiment was performed three times and each sample in each experiment was prepared in duplicate. The level of Rta and RanBPM expression were detected by immunoblotting (IB) with anti-Rta and anti-RanBPM antibodies.

respectively (Fig. 8). The over-expression of two of these three proteins increased the activity of Gal4-Rta by factors of 4.8-6.3 (Fig. 8). The Gal4-Rta activity increased by a factor of 7.5 when Ubc9, SUMO-1 and RanBPM were over-expressed in the cells (Fig. 8), indicating that RanBPM promotes the transactivation activity of Rta by sumoylation.

\section{Activation of the EBV lytic cycle}

It is generally known that Rta is usually not expressed during EBV latency in P3HR1 cells. Transfecting up to $20 \mu \mathrm{g}$ of pEGFP-RanBPM into $5 \times 10^{6}$ of P3HR1 cells had little effect on the expression of Rta; immunoblot analysis with anti-Rta antibody did not detect the expression of Rta at $24 \mathrm{~h}$ after transfection (Fig. 9, lane 3). Transfecting the cells with $0.5 \mu \mathrm{g}$ of pCMV-R, which is about $10 \%$ of the amount that is

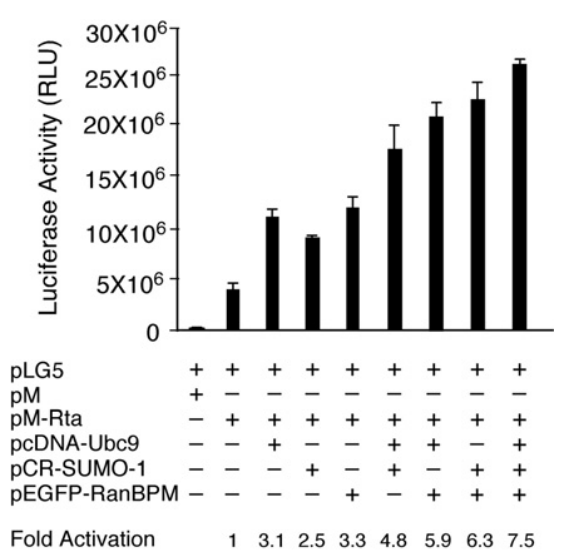

Fig. 8. Enhancement of Rta's transactivation activity by sumoylation enzymes and RanBPM. EBV-negative Akata cells were cotransfected with pcDNA-Ubc9, pCR-SUMO-1, pEGFP-RanBPM, or combinations of these plasmids, to elucidate the effect of sumoylation on the capacity of Gal4Rta to transactivate the pLG5 promoter. Luciferase activity that was exhibited by the cells was examined at $24 \mathrm{~h}$ after transfection. Each transfection experiment was performed at least three times, and each sample in each experiment was prepared in duplicate. RLU: relative light units. typically used in EBV lytic induction, resulted in the synthesis of a small amount of Rta (Fig. 9, lane 4). However, the amount of Rta increased markedly when $5 \mu \mathrm{g}$ or $10 \mu \mathrm{g}$ of pEGFP-RanBPM was also cotransfected (Fig. 9, lanes 5 and 6), indicating that RanBPM promoted the expression of Rta, presumably by autoregulating the BRLF1 promoter. Additionally, the transfection also led to the expression of both phosphorylated and unphosphorylated EA-D (Fig. 9), an early protein of EBV, suggesting that RanBPM affects the lytic development.

\section{Discussion}

Rta and Zta are expressed by EBV during the immediate-early stage of the lytic cycle. ${ }^{36,37}$ These two proteins appear to activate EBV genes in different ways. Transcription of many EBV lytic genes, including those expressed during the immediateearly and early stage of the lytic cycle, seems to depend strongly on Zta. Without Zta, none of these genes is expressed, ${ }^{38-41}$ indicating that Rta alone

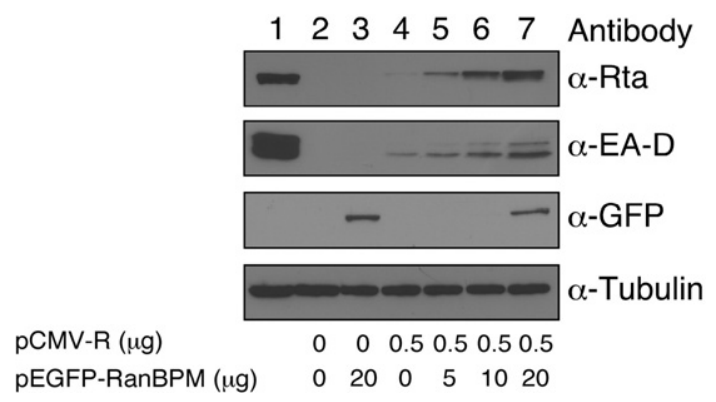

Fig. 9. Effects of RanBPM on EBV lytic activation. P3HR1 cells were treated with $3 \mathrm{mM}$ sodium butyrate to activate the expression of Rta (lane 1). Cells untreated with sodium butyrate (lane 2) were transfected with $20 \mu \mathrm{g}$ of pEGFPRanBPM (lane 3). Cotransfection was also performed by transfecting $0.5 \mu \mathrm{g}$ of pCMV-R and 5-20 $\mu \mathrm{g}$ of pEGFPRanBPM (lanes 4-7). Lysates were prepared at $24 \mathrm{~h}$ after transfection. Immunoblot analysis was conducted using antiRta, add 'anti-EA-D, anti-GFP and anti- $\alpha$-tubulin antibodies. 
does not suffice to complete the EBV lytic cascade. Rta, however, seems to have a different role in activating the EBV lytic genes. Rta binds to RRE in promoters to activate transcription but also interacts indirectly with $\mathrm{Sp} 1$ as a coactivator to enhance the transcription that depends on $\mathrm{Sp} 1,{ }^{12}$ which fact may explain why the transcription of most EBV lytic genes is inefficient without Rta. Since EBV cannot complete its lytic cycle efficiently without Rta, ${ }^{6}$ the capacity of Rta to activate transcription is critical to EBV lytic development. In this work, a yeast twohybrid screen established that RanBPM, a Ran nuclear-cytoplasmic transport protein, also interacts with Rta. The interaction is verified in vitro by GSTpull-down analysis (Fig. 1) and in vivo by coimmunoprecipitation (Fig. 2). This interaction apparently involves a segment of the transactivation domain of Rta, spanning amino acid residues 416-476, and the SPRY domain in RanBPM (Fig. 3). The interaction with RanBPM appears to promote the transactivation activity of Rta in transient transfection analysis (Fig. 7). In fact, RanBPM is known to interact, via its SPRY domain, with many proteins, including TrkA receptor, hDectin-1E, L1,Axl/Sky and hormone receptors, ${ }^{17-20}$ and to affect their biological functions. Since many RanBPM-interacting proteins, including androgen receptor, glucocorticoid receptor, p73 and HIPK2, are conjugated by SUMO, this work investigates whether RanBPM participates in Rta sumoylation. Our previous study demonstrated that Rta is conjugated by SUMO-1 to promote the transactivation activities of Rta. ${ }^{8}$ The work also found that sumoylated Rta was unstable and sensitive to SUMO proteases, causing the detection of sumoylated Rta by immunoblotting difficult. ${ }^{8}$ Therefore, treating cells with $2.5 \mu \mathrm{M}$ MG132 is necessary to stabilize and detect sumoylated Rta by immunoblotting. However, under such experimental conditions, the amount of sumoylated Rta becomes abundant and further enhancement of Rta sumoylation by SUMO E3 ligases was difficult to demonstrate. Therefore, this study reduces the concentration of MG132 to $0.5 \mu \mathrm{M}$ to decrease the amount of detectable sumoylated Rta so that an increase of Rta sumoylation can be detected after over-expressing SUMO E3 ligase. Under the conditions, sumoylated Rta in P3HR1 cells was undetected after cotransfecting pCR-SUMO-1 and pCMV-R (Fig. $5 a$, lane 2 and $b$, lane 3 ). However, over-expression of GFP-RanBPM increases Rta sumoylation (Fig. 5a, lane 3). Previous studies showed that sumoylation efficiency by SUMO E3 ligases is enhanced in the presence of SUMO E2 (Ubc9). ${ }^{42-44}$ In fact, this study find that RanBPM interacts with Ubc9 in vivo (Fig. 6) and over-expression of GFP-RanBPM and Ubc9 profoundly increases the number of sumoylated Rta bands and their intensity in a gel (Fig. 5, lane 6), indicating that RanBPM functions as a SUMO E3 ligase in enhancing the sumoylation of Rta. However, we were unable to demonstrate the same enhancement effect in vitro (data not shown). The lack of enhancement may be attributable to the fact that RanBPM expressed by E. coli is not functional and is incapable of promoting Rta sumoylation. A similar phenomenon was observed by other studies on WRN and p53 sumoylation. ${ }^{45,46}$ We cannot rule out the possibility that, instead of functioning as a SUMO-E3 ligase, RanBPM may actually inhibit the function of SUMO proteases and therefore protect sumoylated Rta from desumoylation. An additional possibility is that RanBPM may recruit Ubc9 and a SUMO E3 ligase to Rta. Our study also found that downregulation of endogenous RanBPM by siRNA did not affect Rta sumoylation (data not shown). Because Rta sumoylation may be enhanced by different SUMO E3 ligases, including PIAS1, PIASx $\alpha$ and PIASx $\beta$, ,27 inhibiting RanBPM expression alone may not be sufficient to inhibit Rta sumoylation. Since sumoylation is known to enhance the transactivation activity of Rta, ${ }^{8,27}$ the fact that RanBPM over-expression promotes Rta sumoylation could explain why the expression of GFP-RanBPM also increases the transactivation activity of Rta (Fig. 7). Additionally, the fact that RanBPM promotes the capacity of Gal4-Rta to transactivate the Gal4 promoter in pLG5 (Fig. 8) further indicates that RanBPM influences Rta's transactivation activity rather than its DNA-binding capacity. The enhanced Rta transactivation activity is less likely due to stabilization of Rta by sumoylation, since Rta levels were not increased by RanBPM overexpression (Fig. 7), although further studies will be necessary to completely rule out that endogeneous Rta becomes more stable in the presence of RanBPM. Most importantly, over-expression of RanBPM and the resulting enhanced Rta activity seem to influence the activation of the EBV lytic cycle by Rta. This study demonstrates that transfection of $0.5 \mu \mathrm{g}$ of pCMV-R, an amount tenfold lower than that typically used for EBV lytic activation, induces production of only a small amount of Rta and EAD. However, cotransfection of pEGFP-RanBPM substantially increases the expression of these two proteins in a dose-dependent manner in P3HR1 cells (Fig. 9). These results clearly indicate the participation of RanBPM in promoting the effect of Rta on EBV lytic activation by Rta.

\section{Materials and Methods}

\section{Cell lines and EBV lytic induction}

P3HR1 and EBV-negative Akata cells were cultured in RPMI 1640 medium containing 10\% (v/v) fetal calf serum. 293T cells were cultured in Dulbecco's modified Eagle's medium (DMEM) that contained 10\% fetal calf serum. To activate the EBV lytic cycle, P3HR1 cells were treated with TPA and sodium butyrate, according to a method described elsewhere. ${ }^{47,48}$

\section{Plasmids}

Plasmid pCMV-R contains BRLF1 that was transcribed from the cytomegalovirus (CMV) immediate-early promoter. Plasmid pCMV-3 is a cloning vector that was used to construct pCMV-R. Plasmid pGEX-4T1, which expresses GST, was purchased from Amersham Biosciences. 
Plasmid pGEX-RanBPM was used to express a GSTRanBPM fusion protein. ${ }^{24}$ Plasmid pEGFP-C1 (Cloetech) was used to construct plasmids that express GFP-fusion proteins. Plasmid pEGFP-Rta was provided by T.-Y. Hsu. ${ }^{49}$ Plasmid pEGFP-RanBPM ${ }^{16}$ expresses a RanBPM-GFP fusion protein. Plasmid pEGFP-RanBPM-209, which encodes deleted RanBPM that lacks the N-terminal 208 amino acids, was constructed by inserting a PCR fragment that was amplified with primers RanBPM-684:

\section{5'-CCCAAGCTTGTCCAGCAGCCTGTGGGATTT}

and RanBPM-3:

\section{5'-CCGCTCGAGTAATGTAGGTAGTCTTCC}

into the HindIII and Sall sites in pEGFP-C1. Plasmid pEGFP-RanBPM-334 was constructed using the same strategy with primers RanBPM-1055:

\section{5'-CCCAAGCTTGTCCTTTCGTGTTTGATATAG}

and RanBPM-3. Plasmid pEGFP-SPRY was constructed in the same way with the DNA fragment that was amplified with primers RanBPM-684 and GST-BPM:

\section{5'-CCGCTCGAGCACGAAAGGATGTTGCCC}

Plasmid pFlag-Rta that expresses full-length Rta with a Flag tag at the amino terminus (Flag-Rta) was constructed by inserting a PCR-amplified BRLF1 fragment that was amplified with primers Flag-5R:

\section{5'-CCGGAATTCCAGGCCTAAAAAGGATGGC}

and GST-3R:

\section{5'-ACGCGTCGACGTCTAAAATAAGCTGGTGTCAA}

into pCMV-Tag2A at the EcoRI and XhoI sites. Plasmids that expressed deletion mutants of Flag-Rta, including R-DN190，R-DN290，R-DN360，R-DN415 and R-DC416, which lacked Rta regions from amino acids 1-190, 1-290, $1-360,1-415$ and 416-605, respectively, were constructed by inserting BRLF1 fragments that had been amplified by PCR, into the EcoRI-XhoI sites in pCMV-Tag2A. Plasmids that expressed deletion mutants of HA-Rta, including R-DC416 and R-DN415, which lack Rta regions from amino acids 416-605 and 1-415, respectively, were constructed by inserting a PCR-amplified BRLF1 fragment into the EcoRI-XhoI sites in pcDNA3-HA. Plasmids pRRE, pBMLF1-luc, pSp1-luc and pp21-luc were reporter plasmids that were used to analyze the transactivation activation activity of Rta. ${ }^{27}$ Plasmid pLG5 has five Gal4binding sites and a TATA sequence upstream of a luciferase gene. Plasmid $\mathrm{pM}$ is an empty vector that contains the GAL4 gene (Clontech). A DNA fragment that encodes an Rta region from amino acids 255-605 was amplified by PCR and inserted into the EcoRI and SalI sites in $\mathrm{pM}$ to generate $\mathrm{pM}-\mathrm{R}$. Plasmids pCR-SUMO-1 and pcDNA-Ubc9 expressed Flag-tagged SUMO-1 and HAtagged Ubc9, respectively.

\section{Yeast two-hybrid screen}

Yeast two-hybrid screening of the binding partners of Rta was performed with a bait plasmid, pR476 as described. ${ }^{8,27}$

\section{GST pull-down assays}

GST pull-down assays with purified GST-RanBPM were performed as described. ${ }^{8}$

\section{Immunoprecipitation}

293 T cells $\left(1 \times 10^{7}\right)$ were transfected with pCMV-R, pEGFP-RanBPM or pcDNA-Ubc9 by electroporation. ${ }^{50}$ Cell lysate was prepared $24 \mathrm{~h}$ after transfection and mixed with anti-HA (Roche Applied Science, Penzberg, Germany), anti-GFP (Santa Cruz Biotech, Santa Cruz, CA) or anti-Rta (Argene, Varilhes, France) antibody. For endogenous interaction between Rta and RanBPM, P3HR1 cells were induced with TPA and sodium butyrate for $24 \mathrm{~h}$ and cell lysate was mixed with anti-Rta or anti-RanBPM antibody. ${ }^{24}$ Proteins that had been immunoprecipitated by the antibodies were captured with protein A/G-agarose beads (Oncogene, Boston, MA). Immunoblot analysis was performed to detect immunoprecipitated proteins. Sumoylated Rta was detected as described. ${ }^{51}$

\section{Immunoblot analysis}

Proteins that were separated by SDS-PAGE were electrotransferred to Hybond $\mathrm{C}$ membrane (Amersham Biosciences, New Jersey) at $90 \mathrm{~V}$ for $1 \mathrm{~h}$, and probed using appropriate antibodies. Supersignal West Pico chemiluminescent substrate (Pierce, Rockford, IL) was used to visualize the proteins on the membrane. The antibodies used included anti-Rta, anti-HA, anti-GFP, anti- $\alpha$-tubulin (Sigma, St Louis, MO) and anti-Flag antibodies (Sigma). AntiRanBPM monoclonal antibody is described elsewhere. ${ }^{24}$

\section{Transient transfection assay}

P3HR1, EBV-negative Akata and 293T cells $\left(5 \times 10^{6}\right)$ were transfected with $5 \mu \mathrm{g}$ of plasmids by electroporation using a BTX ECM630 electroporator (BTX Instrument, Holliston, MA) and luciferase assay was performed using a luminometer (Orion II; Berthod, Bad Wildbad, Germany) as described. ${ }^{50}$ Each transfection experiment was performed three times and each sample in each experiment was prepared in duplicate.

\section{Immunofluorescence analysis}

P3HR1 cells were cultured for $24 \mathrm{~h}$ after transfecting pEGFP, pEGFP-Rta, pCMV-3, pHA-R-DC416, or pHA-RDN415. Cells were harvested by centrifugation; plated on poly-l-lysine (Sigma)-coated coverslips, and fixed with $4 \%$ $(\mathrm{v} / \mathrm{v})$ paraformaldehyde in phosphate-buffered saline (PBS) for $30 \mathrm{~min}$. The cells were then incubated for $1 \mathrm{~h}$ with anti-RanBPM monoclonal antibody, rabbit anti-HA polyclonal antibody (Y-11, Santa Cruz Biotechnology Inc., Santa Cruz, CA), or goat anti-Ubc9 polyclonal antibody (N-15, Santa Cruz Biotechnology Inc.). Following incubation, cells were washed three times in PBS and then treated with fluorescein Alexa 488-conjugated goat anti-mouse IgG antibodies (Molecular Probes, Inc. Eugene, OR), Alexa 598-conjugated goat anti-rabbit IgG antibodies (Molecular Probes), or rhodamine-conjugated donkey anti-goat IgG antibodies. Nuclei were visualized by staining with $5 \mu \mathrm{g} /$ $\mathrm{ml}$ of 4'-6-diamidino-2-phenylindole. Finally, cells were washed in PBS, mounted in CITIFLOUR (Agar Inc. Essex, England), and observed with a Zeiss confocal laser scanning microscope (META, model LSM 510). 


\section{Acknowledgements}

Dr Takeharu Nishimoto in Kyushu University is appreciated for providing an RanBPM plasmid. Dr. T.-Y. Hsu is appreciated for providing pEGFP-Rta. This research was supported by the National Health Research Institute of the Republic of China, Taiwan (contract no. NHRI-EX95-9503BC) and the National Science Council of the Republic of China, Taiwan (contract no. NSC95-3112-B-182-002).

\section{References}

1. Kieff, E. (1996). Epstein-Barr virus and its replication. In Fields' Virology (Fields, B. N., Knipe, D. M. \& Howley, P. M., eds), 3rd edit. Lippincott-Raven, Philadelphia, PA.

2. Speck, S. H., Chatila, T. \& Flemington, E. (1997). Reactivation of Epstein-Barr virus: regulation and function of the BZLF1 gene. Trends Microbiol. 5, 399-405.

3. Liu, P. \& Speck, S. H. (2003). Synergistic autoactivation of the Epstein-Barr virus immediate-early BRLF1 promoter by Rta and Zta. Virology, 310, 199-206.

4. Holley-Guthrie, E. A., Quinlivan, E. B., Mar, E. C. \& Kenney, S. (1990). The Epstein-Barr virus (EBV) BMRF1 promoter for early antigen (EA-D) is regulated by the EBV transactivators, BRLF1 and BZLF1, in a cell-specific manner. J. Virol. 64, 3753-3759.

5. Gruffat, H., Duran, N., Buisson, M., Wild, F., Buckland, R. \& Sergeant, A. (1992). Characterization of an Rbinding site mediating the R-induced activation of the Epstein-Barr virus BMLF1 promoter. J. Virol. 66, 46-52.

6. Feederle, R., Kost, M., Baumann, M., Janz, A., Drouet, E., Hammerschmidt, W. \& Delecluse, H. J. (2000). The Epstein-Barr virus lytic program is controlled by the co-operative functions of two transactivators. EMBO J. 19, 3080-3089.

7. Gruffat, H., Manet, E., Rigolet, A. \& Sergeant, A (1990). The enhancer factor $\mathrm{R}$ of Epstein-Barr virus (EBV) is a sequence-specific DNA binding protein Nucleic Acids Res. 18, 6835-6843.

8. Chang, L. K., Lee, Y. H., Cheng, T. S., Hong, Y. R., Lu, P. J., Wang, J. J. et al. (2004). Post-translational modification of Rta of Epstein-Barr virus by SUMO-1. J. Biol. Chem. 279, 38803-38812.

9. Swenson, J. J., Holley-Guthrie, E. \& Kenney, S. C. (2001) Epstein-Barr virus immediate-early protein BRLF1 interacts with CBP, promoting enhanced BRLF1 transactivation. J. Virol. 75, 6228-6234.

10. Zacny, V. L., Wilson, J. \& Pagano, J. S. (1998). The Epstein-Barr virus immediate-early gene product, BRLF1, interacts with the retinoblastoma protein during the viral lytic cycle. J. Virol. 72, 8043-8051.

11. Swenson, J. J., Mauser, A. E., Kaufmann, W. K. \& Kenney, S. C. (1999). The Epstein-Barr virus protein BRLF1 activates $S$ phase entry through E2F1 induction. J. Virol. 73, 6540-6550.

12. Chang, L. K., Chung, J. Y., Hong, Y. R., Ichimura, T., Nakao, M. \& Liu, S. T. (2005). Activation of Sp1mediated transcription by Rta of Epstein-Barr virus via an interaction with MCAF1. Nucleic Acids Res. 33, 6528-6539.

13. Adamson, A. L., Darr, D., Holley-Guthrie, E., Johnson, R. A., Mauser, A., Swenson, J. \& Kenney, S. (2000). Epstein-Barr virus immediate-early proteins BZLF1 and BRLF1 activate the ATF2 transcription factor by increasing the levels of phosphorylated p38 and c-Jun N-terminal kinases. J. Virol. 74, 1224-1233.

14. Chua, H. H., Lee, H. H., Chang, S. S., Lu, C. C., Yeh, T. H., Hsu, T. Y. et al. (2007). Role of the TSG101 gene in Epstein-Barr virus late gene transcription. J. Virol. 81, 2459-2471.

15. Nakamura, M., Masuda, H., Horii, J., Kuma, K., Yokoyama, N., Ohba, T. et al. (1998). When overexpressed, a novel centrosomal protein, RanBPM, causes ectopic microtubule nucleation similar to gammatubulin. J. Cell Biol. 143, 1041-1052.

16. Nishitani, H., Hirose, E., Uchimura, Y., Nakamura, M., Umeda, M., Nishii, K. et al. (2001). Full-sized RanBPM cDNA encodes a protein possessing a long stretch of proline and glutamine within the N-terminal region, comprising a large protein complex. Gene, 272, 25-33.

17. Yuan, Y., Fu, C., Chen, H., Wang, X., Deng, W. \& Huang, B. R. (2006). The Ran binding protein RanBPM interacts with TrkA receptor. Neurosci. Lett. 407, 26-31.

18. Xie, J., Sun, M., Guo, L., Liu, W., Jiang, J., Chen, X. et al. (2006). Human Dectin-1 isoform E is a cytoplasmic protein and interacts with RanBPM. Biochem. Biophys. Res. Commun. 347, 1067-1073.

19. Cheng, L., Lemmon, S. \& Lemmon, V. (2005). RanBPM is an L1-interacting protein that regulates L1mediated mitogen-activated protein kinase activation. J. Neurochem. 94, 1102-1110.

20. Hafizi, S., Gustafsson, A., Stenhoff, J. \& Dahlback, B. (2005). The Ran binding protein RanBPM interacts with Axl and Sky receptor tyrosine kinases. Int. J. Biochem. Cell. Biol. 37, 2344-2356.

21. Rao, M. A., Cheng, H., Quayle, A. N., Nishitani, H., Nelson, C. C. \& Rennie, P. S. (2002). RanBPM, a nuclear protein that interacts with and regulates transcriptional activity of androgen receptor and glucocorticoid receptor. J. Biol. Chem. 277, 48020-48027.

22. Poirier, M. B., Laflamme, L. \& Langlois, M. F. (2006). Identification and characterization of RanBPM, a novel coactivator of thyroid hormone receptors. J. Mol. Endocrinol. 36, 313-325.

23. Wang, D., Li, Z., Messing, E. M. \& Wu, G. (2002). Activation of Ras/Erk pathway by a novel MET-interacting protein RanBPM. J. Biol. Chem. 277, 36216-36222.

24. Denti, S., Sirri, A., Cheli, A., Rogge, L., Innamorati, G., Putignano, S. et al. (2004). RanBPM is a phosphoprotein that associates with the plasma membrane and interacts with the integrin LFA-1. J. Biol. Chem. 279, 13027-13034.

25. Bai, D., Chen, H. \& Huang, B. R. (2003). RanBPM is a novel binding protein for p75NTR. Biochem. Biophys. Res. Commun. 309, 552-557.

26. Kramer, S., Ozaki, T., Miyazaki, K., Kato, C., Hanamoto, T. \& Nakagawara, A. (2005). Protein stability and function of p73 are modulated by a physical interaction with RanBPM in mammalian cultured cells. Oncogene, 24, 938-944.

27. Liu, S. T., Wang, W. H., Hong, Y. R., Chuang, J. Y., Lu, P. J. \& Chang, L. K. (2006). Sumoylation of Rta of Epstein-Barr virus is preferentially enhanced by PIASxbeta. Virus Res. 119, 163-170.

28. Ponting, C., Schultz, J. \& Bork, P. (1997). SPRY domains in ryanodine receptors $(\mathrm{Ca}(2+)$-release channels). Trends Biochem. Sci. 22, 193-194.

29. Poukka, H., Karvonen, U., Janne, O. A. \& Palvimo, J. J. (2000). Covalent modification of the androgen receptor by small ubiquitin-like modifier 1 (SUMO-1). Proc. Natl Acad. Sci. USA, 97, 14145-14150.

30. Le Drean, Y., Mincheneau, N., Le Goff, P. \& Michel, D. (2002). Potentiation of glucocorticoid receptor trans- 
criptional activity by sumoylation. Endocrinology, 143, 3482-3489.

31. Tian, S., Poukka, H., Palvimo, J. J. \& Janne, O. A (2002). Small ubiquitin-related modifier-1 (SUMO-1) modification of the glucocorticoid receptor. Biochem. J. 367, 907-911.

32. Kim, Y. H., Choi, C. Y. \& Kim, Y. (1999). Covalent modification of the homeodomain-interacting protein kinase 2 (HIPK2) by the ubiquitin-like protein SUMO1. Proc. Natl Acad. Sci. USA, 96, 12350-12355.

33. Minty, A., Dumont, X., Kaghad, M. \& Caput, D. (2000). Covalent modification of p73alpha by SUMO1. Two-hybrid screening with p73 identifies novel SUMO-1-interacting proteins and a SUMO-1 interaction motif. J. Biol. Chem. 275, 36316-36323.

34. Bailey, D. \& O'Hare, P. (2005). Comparison of the SUMO1 and ubiquitin conjugation pathways during the inhibition of proteasome activity with evidence of SUMO1 recycling. Biochem. J. 392, 271-281.

35. Roukens, M. G., Alloul-Ramdhani, M., Vertegaal, A. C., Anvarian, Z., Balog, C. I., Deelder, A. M. et al. (2008). Identification of a new site of sumoylation on Tel (ETV6) uncovers a PIAS-dependent mode of regulating Tel function. Mol. Cell. Biol. 28, 2342-2357.

36. Biggin, M., Bodescot, M., Perricaudet, M. \& Farrell, P. (1987). Epstein-Barr virus gene expression in P3HR1superinfected Raji cells. J. Virol. 61, 3120-3132.

37. Takada, K. \& Ono, Y. (1989). Synchronous and sequential activation of latently infected Epstein-Barr virus genomes. J. Virol. 63, 445-449.

38. Buisson, M., Manet, E., Trescol-Biemont, M. C., Gruffat, H., Durand, B. \& Sergeant, A. (1989). The Epstein-Barr virus (EBV) early protein EB2 is a posttranscriptional activator expressed under the control of EBV transcription factors EB1 and R. J. Virol. 63, 5276-5284.

39. Cox, M. A., Leahy, J. \& Hardwick, J. M. (1990). An enhancer within the divergent promoter of EpsteinBarr virus responds synergistically to the $\mathrm{R}$ and $\mathrm{Z}$ transactivators. J. Virol. 64, 313-321.

40. Kenney, S., Holley-Guthrie, E., Mar, E. C. \& Smith, M. (1989). The Epstein-Barr virus BMLF1 promoter contains an enhancer element that is responsive to the BZLF1 and BRLF1 transactivators. J. Virol. 63, 3878-3883.
41. Kenney, S., Kamine, J., Holley-Guthrie, E., Lin, J. C., Mar, E. C. \& Pagano, J. (1989). The Epstein-Barr virus (EBV) BZLF1 immediate-early gene product differentially affects latent versus productive EBV promoters. J. Virol. 63, 1729-1736.

42. Kagey, M. H., Melhuish, T. A. \& Wotton, D. (2003). The polycomb protein Pc2 Is a SUMO E3. Cell, 113, 127-137.

43. Ji, Z., Degerny, C., Vintonenko, N., Deheuninck, J., Foveau, B., Leroy, C. et al. (2007). Regulation of the Ets-1 transcription factor by sumoylation and ubiquitinylation. Oncogene, 26, 395-406.

44. Buschmann, T., Lerner, D., Lee, C. G. \& Ronai, Z. (2001). The Mdm-2 amino terminus is required for Mdm2 binding and SUMO-1 conjugation by the E2 SUMO-1 conjugating enzyme Ubc9. J. Biol. Chem. 276, 40389-40395.

45. Woods, Y. L., Xirodimas, D. P., Prescott, A. R., Sparks, A., Lane, D. P. \& Saville, M. K. (2004). p14 Arf promotes small ubiquitin-like modifier conjugation of Werners helicase. J. Biol. Chem. 279, 50157-50166.

46. Chen, L. \& Chen, J. (2003). MDM2-ARF complex regulates p53 sumoylation. Oncogene, 22, 5348-5357.

47. Luka, J., Kallin, B. \& Klein, G. (1979). Induction of the Epstein-Barr virus (EBV) cycle in latently infected cells by n-butyrate. Virology, 94, 228-231.

48. Davies, A. H., Grand, R. J., Evans, F. J. \& Rickinson, A. B. (1991). Induction of Epstein-Barr virus lytic cycle by tumor-promoting and non-tumor-promoting phorbol esters requires active protein kinase C. J. Virol. 65, 6838-6844.

49. Hsu, T. Y., Chang, Y., Wang, P. W., Liu, M. Y., Chen, M. R., Chen, J. Y. \& Tsai, C. H. (2005). Reactivation of Epstein-Barr virus can be triggered by an Rta protein mutated at the nuclear localization signal. J. Gen. Virol. 86, 317-322.

50. Chang, P. J., Chang, Y. S. \& Liu, S. T. (1998). Role of Rta in the translation of bicistronic BZLF1 of Epstein-Barr virus. J. Virol. 72, 5128-5136.

51. Sapetschnig, A., Rischitor, G., Braun, H., Doll, A., Schergaut, M., Melchior, F. \& Suske, G. (2002). Transcription factor Sp3 is silenced through SUMO modification by PIAS1. EMBO J. 21, 5206-5215. 